\title{
Analysis of changes in intestinal flora and intravascular inflammation and coronary heart disease in obese patients
}

\author{
XV LI and CHUANTAO LI \\ Department of Internal Medicine, Maternity and Child Health Care of Zaozhuang, \\ Zaozhuang, Shandong 277100, P.R. China
}

Received November 14, 2017; Accepted January 11, 2018

DOI: $10.3892 /$ etm.2018.5987

\begin{abstract}
Changes in intestinal flora in obese patients and intravascular C-reactive protein (CRP), interleukin-6 (IL-6) and tumor necrosis factor- $\alpha(\mathrm{TNF}-\alpha)$ and coronary heart disease (CHD) were analyzed. A total of 75 cases of obese patients were divided into obesity $(\mathrm{OB})$ alone $(\mathrm{n}=40)$ and $\mathrm{OB}$ with CHD group $(n=35)$. There was no statistically significant difference in age, sex, pre-existing basic diabetes, history of hypertension, and body mass index $(\mathrm{P}>0.05)$. Results showed that total bacterial load of CHD was obviously higher than that of $\mathrm{OB}$ group. The uric acid decomposed by intestinal flora (IFUA) and blood uric acid levels in CHD were higher than those in OB group, but the fecal uric acid level was lower than that of $\mathrm{OB}$ group $(\mathrm{P}<0.05)$. Levels of inflammatory factors in $\mathrm{CHD}$, were significantly higher than those in OB group $(\mathrm{P}<0.05)$. Correlation analyses showed that the intestinal flora total load and CRP were positively correlated $(r=0.793$, $\mathrm{P}<0.001)$. Intestinal flora and Gensini score were also positively related to total load $(r=0.893, P=0.893)$. Furthermore, CRP and Gensini score were positively related $(r=0.796$, $\mathrm{P}<0.796)$. IFUA and Gensini score were positively related to $(\mathrm{r}=0.647, \mathrm{P}<0.001)$. Over-reaction in the flammation system in obese patients may lead to intestinal flora disorder, disturbance and also increased levels of IFUA and inflammatory factors.
\end{abstract}

\section{Introduction}

The human intestine is also known as the second brain, on the surface of which numerous intestinal flora play an important role, impacting on the body's immune system, endocrine system and spiritual emotion; most importantly, it is closely

Correspondence to: Dr Chuantao $\mathrm{Li}$, Department of Internal Medicine, Maternity and Child Health Care of Zaozhuang, 25 Wenhua East Road, Zaozhuang, Shandong 277100, P.R. China E-mail: 1ct198964@163.com

Key words: obesity, flora disorder, coronary heart disease, inflammatory cytokines related to digestive diseases (1). However, it has been found in previous studies that intestinal flora disturbance may lead to the occurrence or aggravation of coronary heart disease (CHD) to a certain extent (2). In patients with CHD, the total amount of bacteria in the intestine is increased, but the content of probiotics is reduced and the content of harmful strains is increased; in particular, intestinal flora disturbance in obese patients is more likely to become a risk factor for CHD (3). In healthy people, intestinal flora is relatively stable, because the mucus produced by the intestinal mucosa contains a certain amount of antimicrobial peptides, which are a kind of protein that prevents the overgrowth of intestinal flora. At the same time, the secretion and expression of antimicrobial peptides are inhibited when the number of intestinal flora is too low (4). When the body's homeostasis is damaged, the intestinal flora species and the level of antibacterial peptides will change, leading to the occurrence of intestinal flora disturbance and a variety of related diseases (5). Compared with healthy people, obese patients are more prone to intestinal flora disturbance (6), of which the most representative is the decreased proportion of Bacteroidetes, the increased proportion of phylum Firmicutes and over-metabolism of intestinal Clostridium, causing energy metabolism disorder and obesity (OB)-related metabolic diseases (7). On the other hand, when intestinal flora disturbance occurs, the immune system will be destroyed, the harmful strains in the intestine can secrete endotoxins and so on, and the intestinal mucosa will be damaged, causing excessive intravascular inflammatory response, elevated levels of inflammatory factors in serum, along with increased expression of adhesion molecules, disorders of vascular regulatory factor secretion and endothelial damage, eventually leading to atherosclerosis (8). Therefore, to explore the relationship of the intestinal flora changes and inflammatory factors in obese patients with CHD can effectively reduce the incidence rate of CHD in obese patients and provide more treatment methods for patients with $\mathrm{CHD}$.

\section{Patients and methods}

General data. A total of 75 obese patients in Child Health Care of Zaozhuang Hospital from March 2015 to September 2016 were selected, and all selected patients with body mass index 
Table I. Gensini score calculation.

\begin{tabular}{lcll}
\hline Angiostegnosis & Score & \multicolumn{1}{c}{ Diseased artery } & Score \\
\hline$<25 \%$ & 1 & Left main & \\
$25-50 \%$ & 2 & Left descending branch or proximal circumflex artery & 5 \\
$51-75 \%$ & 4 & Middle left anterior descending branch & 2.5 \\
$76-90 \%$ & 8 & Distal left anterior descending branch anterior & 1.5 \\
$91-99 \%$ & 16 & Middle and distal left circumflex artery & 1 \\
$100 \%$ & 32 & Right coronary artery, small branch & 1 \\
\end{tabular}

(BMI) of $\geq 28 \mathrm{~kg} / \mathrm{m}^{2}$, and the degree of arterial stenosis above $70-75 \%$ according to the patient's clinical manifestations, electrocardiogram or coronary arteriongraphy were diagnosed with $\mathrm{CHD}$. They were divided into $\mathrm{OB}$ alone $(\mathrm{n}=40)$ and $\mathrm{OB}$ with CHD group, including 53 males and 22 females aged 32-75 years with an average age of $(46.73 \pm 5.32)$ years. The present study was approved by the Ethics Committee of Maternity and Child Health Care of Zaozhuang (Zaozhuang, China). Signed informed consents were obtained from all participants before the study. Exclusion criteria; patients with previous history of chronic diseases, such as hypertension or diabetes; with severe infectious diseases; with diseases related to immune dysfunction; with cardiac status and myocardial diseases; with severe valvular heart disease; and patients who did not use probiotics, antibiotics or other related drugs that affected the intestinal flora prior to inclusion into the experiment.

\section{Methods}

Detection of total intestinal flora load and main strains. The feces of the included patients were collected, and $350 \mathrm{~g}$ of the middle part of the feces was selected as the final specimen. The SYBR-Green I fluorescence quantitative polymerase chain reaction (PCR) was used to determine the total load of all bacteria and the main strain load in the feces, including two probiotic strains, Bifidobacteria and Lactobacilli, and six kinds of harmful bacteria, Escherichia coli, Helicobacter pylori, Streptococcus, Staphylococcus, Pseudomonas aeruginosa and Veillonella. The strain deoxyribonucleic acid was extracted using the kit provided by Shandong Science and Technology Co., Ltd., (Shandong, China) and the PCR was carried out. Finally, the results were measured by the Light Cycler PCR analyzer (Roche Molecular Diagnostics, Pleasanton, CA, USA). The logarithm of the fecal bacterial copy number / gram represented the intestinal bacterial load.

Detection of fecal uric acid content. The middle part of the feces was centrifuged several times, and the supernatant was taken in the colorimetric cup to detect the fecal uric acid (FUA) content by enzyme colorimetric assay. The results were expressed by the uric acid content decomposed by the intestinal flora (IFUA) in one gram of feces and the original FUA content.

Detection of levels of blood uric acid and inflammatory factors. Peripheral blood $(20 \mathrm{ml})$ was extracted from the included patients after $10 \mathrm{~h}$ of fasting and water deprivation overnight, and the upper serum was taken. Serum $(8 \mathrm{ml})$ was used to determine $\mathrm{C}$-reactive protein (CRP), interleukin-6 (IL-6) and tumor necrosis factor- $\alpha$ (TNF- $\alpha$ ) by immunoturbidimetry, with the reagents and instruments provided by Shandong Biological Instrument Company (Jinan, China), and the remaining $12 \mathrm{ml}$ was used to detect blood uric acid (BUA) level by phosphotungstic acid method with 8,100 automatic biochemical analyzer.

The diagnostic criteria for coronary artery stenosis. Any one lesion in circumflex branch of left coronary artery, left anterior descending and right coronary artery indicated the single- any two lesions indicated the double-, and all lesions indicated the triple-vessel lesion; moreover, when the left main coronary artery lesion occurred, it was also the double-vessel lesion whether or not complicated with other branch lesions; when the left main coronary artery lesion was combined with the right coronary artery lesion, it was also the triple-vessel lesion; Gensini score was calculated (Table I).

Statistical analysis. The data were processed by SPSS 19.0 (IBM Corp., Armonk, NY, USA), collection data are expressed as mean $\pm \mathrm{SD}$, enumeration data were compared by $\chi^{2}$ test, and correlation analysis was used for two factors. $\mathrm{P}<0.05$ was considered to indicate a statistically significant difference.

\section{Results}

Comparison of general data between the $C H D$ and $O B$ group. There were no statistically significant differences in age, sex, previous history of basic diabetes and hypertension, and BMI between the CHD and the OB group ( $\mathrm{P}>0.05)$, and the data were comparable (Table II).

Comparisons of total bacterial and main strain load between the CHD and the $O B$ group. The total bacterial load in the CHD was significantly higher than that in the OB group. The levels of two probiotic strains, Bifidobacterium and Lactobacillus, were significantly lower than those in the OB group, but the levels of harmful strains, including Escherichia coli, Helicobacter pylori, Streptococcus and Staphylococcus, were significantly higher than those in the OB group, and the differences were statistically significant $(\mathrm{P}<0.05)$; but there were no differences in Pseudomonas aeruginosa and Veillonella $(\mathrm{P}>0.05)$ (Table III).

Comparison of the levels of FUA and BUA between the CHD and the $O B$ group. The levels of IFUA and BUA in the CHD 
Table II. Comparisons of general data between the CHD and the OB group.

\begin{tabular}{lccc}
\hline & \multicolumn{2}{c}{ Groups } \\
\cline { 2 - 3 } General data & $\begin{array}{c}\text { CHD } \\
(\mathrm{n}=35)\end{array}$ & $\begin{array}{c}\text { OB } \\
(\mathrm{n}=40)\end{array}$ & P-value \\
\hline Age (years) & $47.61 \pm 4.99$ & $45.82 \pm 5.28$ & 0.873 \\
Sex (male/female) & $25 / 10$ & $28 / 12$ & 0.798 \\
Diabetes & 0 & 0 & 0.916 \\
Hypertension & 0 & 0 & 0.916 \\
BMI $\left(\mathrm{kg} / \mathrm{m}^{2}\right)$ & $29.42 \pm 3.96$ & $28.96 \pm 4.70$ & 0.577 \\
\hline
\end{tabular}

BMI, body mass index; CHD, coronary heart disease; OB, obesity.

Table III. Comparisons of total bacterial load and main strain load between the CHD and the OB group.

\begin{tabular}{lccc}
\hline & \multicolumn{2}{c}{ Groups } & \\
\cline { 2 - 3 } Bacterial load & $\begin{array}{c}\text { CHD } \\
(\mathrm{n}=35)\end{array}$ & $\begin{array}{c}\text { OB } \\
(\mathrm{n}=40)\end{array}$ & P-value \\
\hline Total bacterial load & 12.03 & 7.79 & 0.001 \\
Bifidobacteria & 2.98 & 6.82 & $>0.05$ \\
Lactobacillus & 1.29 & 5.41 & 0.005 \\
Escherichia coli & 8.96 & 5.42 & 0.019 \\
Helicobacter pylori & 9.41 & 6.89 & 0.017 \\
Streptococcus & 8.05 & 3.92 & 0.001 \\
Staphylococcus & 6.43 & 3.06 & 0.047 \\
Pseudomonas aeruginosa & 5.71 & 6.38 & 0.072 \\
Veillonella & 5.23 & 5.17 & 0.083 \\
\hline
\end{tabular}

CHD, coronary heart disease; OB, obesity.

Table IV. Comparisons of the levels of FUA and BUA between the CHD and the OB group.

\begin{tabular}{lccr}
\hline & \multicolumn{3}{c}{ Groups } \\
\cline { 2 - 3 } Uric acid level & $\begin{array}{c}\text { CHD } \\
(\mathrm{n}=35)\end{array}$ & $\begin{array}{c}\text { OB } \\
(\mathrm{n}=40)\end{array}$ & P-value \\
\hline IFUA $(\mathrm{mg} / \mathrm{g})$ & $0.42 \pm 0.15$ & $0.15 \pm 0.09$ & 0.001 \\
FUA $(\mu \mathrm{mol} / \mathrm{l})$ & $13.93 \pm 7.97$ & $19.08 \pm 4.93$ & 0.001 \\
BUA $(\mu \mathrm{mol} / \mathrm{l})$ & $492.92 \pm 75.41$ & $301.53 \pm 49.83$ & 0.001 \\
\hline
\end{tabular}

IFUA, uric acid decomposed by the flora in $1 \mathrm{~g}$ feces (mg); FUA, fecal uric acid $(\mu \mathrm{mol} / \mathrm{l})$; BUA, blood uric acid.

were higher than those in the OB group, but the FUA content was lower than that in the OB group $(\mathrm{P}<0.05)$ (Table IV). Comparison of levels of inflammatory factors between the CHD and the OB group. The levels of peripheral blood inflammatory factors in the
Table V. Comparison of the levels of inflammatory factors between the CHD and the OB group.

\begin{tabular}{lccc}
\hline \multicolumn{3}{c}{ Groups } \\
\cline { 2 - 3 } Inflammatory \\
\cline { 2 - 3 } factors & $\begin{array}{c}\text { CHD } \\
(\mathrm{n}=35)\end{array}$ & $\begin{array}{c}\text { OB } \\
(\mathrm{n}=40)\end{array}$ & P-value \\
\hline $\mathrm{TNF}-\alpha(\mathrm{pg} / \mathrm{ml})$ & $14.29 \pm 4.18$ & $8.64 \pm 2.29$ & 0.001 \\
$\mathrm{IL}-6(\mathrm{ng} / \mathrm{l})$ & $17.43 \pm 2.23$ & $8.41 \pm 1.16$ & 0.001 \\
$\mathrm{CRP}(\mathrm{mg} / \mathrm{l})$ & $8.42 \pm 1.44$ & $2.81 \pm 1.68$ & 0.001 \\
\hline
\end{tabular}

CHD, coronary heart disease; OB, obesity; TNF- $\alpha$, tumor necrosis factor- $\alpha$; IL-6, interleukin-6; CRP, C-reactive protein.

Table VI. Comparison of the coronary artery lesions and Gensini score between the CHD and the OB group.

\begin{tabular}{lccr}
\hline & \multicolumn{2}{c}{ Groups } & \\
\cline { 2 - 3 } Lesion & $\begin{array}{c}\text { CHD } \\
(\mathrm{n}=35)\end{array}$ & $\begin{array}{c}\text { OB } \\
(\mathrm{n}=40)\end{array}$ & P-value \\
\hline Single-vessel lesion & $14.93 \%$ & $51.19 \%$ & 0.001 \\
Double-vessel lesion & $30.66 \%$ & $18.25 \%$ & 0.029 \\
Triple-vessel lesion & $54.41 \%$ & $30.56 \%$ & 0.021 \\
Gensini score (points) & $36.26 \pm 19.38$ & $9.69 \pm 15.87$ & $>0.05$ \\
\hline CHD, coronary heart disease; OB, obesity. & & \\
\hline
\end{tabular}

CHD group, including CRP, IL-6 and TNF- $\alpha$, were significantly higher than those in the $\mathrm{OB}$ group $(\mathrm{P}<0.05)$ (Table $\mathrm{V})$.

Comparison of the coronary artery lesions and Gensini score between the $\mathrm{CHD}$ and the $\mathrm{OB}$ group. By comparison of two groups of patients, it was found that the incidence rate of single coronary artery lesion (14.93\%) in the CHD group was lower than that in patients without CHD (51.19\%), but the incidence rate of double- and triple-vessel lesions were significantly higher than those in patients without CHD (30.66 and $56.15 \%)$, and the differences were statistically significant $(\mathrm{P}<0.05)$ (Table VI).

Correlation analysis. The total intestinal flora load was positively correlated with CRP $(r=0.793, \mathrm{P}>0.05)$ and Gensini score $(\mathrm{r}=0.893, \mathrm{P}>0.05), \mathrm{CRP}$ was positively correlated with Gensini score $(r=0.796, P<0.001)$, and the activity of FUA decomposed by intestinal flora was positively correlated with Gensini score $(\mathrm{r}=0.647, \mathrm{P}<0.001)$ (Figs. 1-4).

\section{Discussion}

The process of maintaining human life and health, the stability of intestinal flora plays a significant role, and intestinal flora disturbance not only leads to the occurrence of digestive diseases (9), but also affects the occurrence and development of other diseases of the body, such as metabolic, immune and 


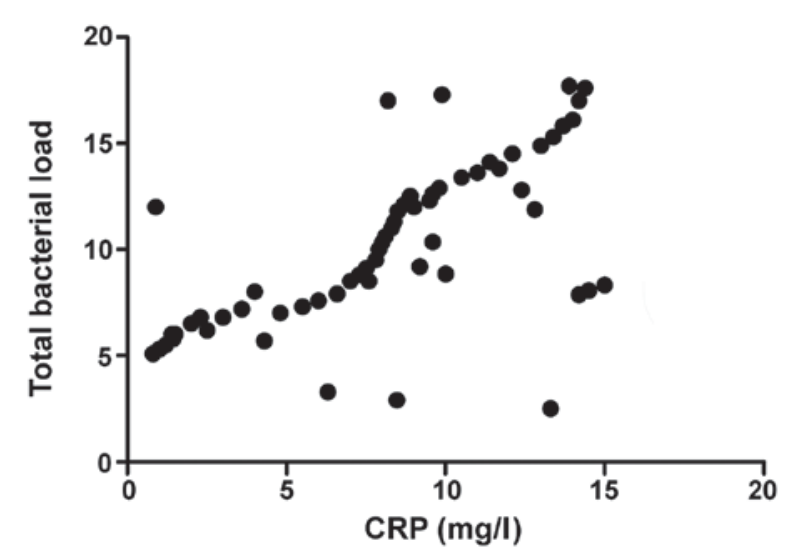

Figure 1. Correlation analysis between CRP and total bacterial load. CRP, C-reactive protein.

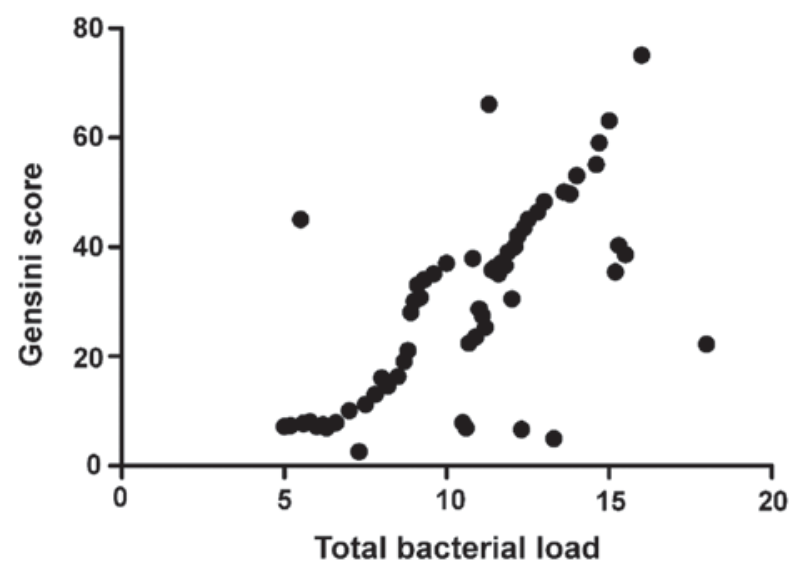

Figure 2. Correlation analysis between total bacterial load and Gensini score.

cardiovascular system diseases, and also affects the changes in brain emotion (10). In healthy people, intestinal flora is in a state of balance, which can protect the body, promote the digestion and absorption of food, and maintain the homeostasis of the internal environment. When the intestinal flora disturbance occurs, it will cause various diseases of the body (11). Increasing experimental data show that intestinal flora disturbance in obese patients is related to the occurrence of CHD (12). It has been found that the increase of intestinal pathogens is related to cardiac risk events and the severity and prognosis of disease, and a variety of harmful strains in the intestine may play a superimposed effect (13). When the total load of bacteria and harmful bacteria in the intestine is increased, and the number of probiotics is reduced, it will aggravate the overreaction of inflammatory stress and increase the possibility of coronary atherosclerotic heart disease (14). It was also found in the present study that the total bacterial load and harmful bacteria load in the CHD were significantly higher than those in the OB group, the differences were statistically significant $(\mathrm{P}<0.05)$, and the total bacterial load was positively correlated with the Gensini score. In addition, most of the clinical views believe that the occurrence of cardiovascular disease is related to the elevated BUA level (15). When intestinal flora disturbance occurs, the increased harmful bacteria will lead to the increased level of FUA decomposed by bacteria, decreased level of original

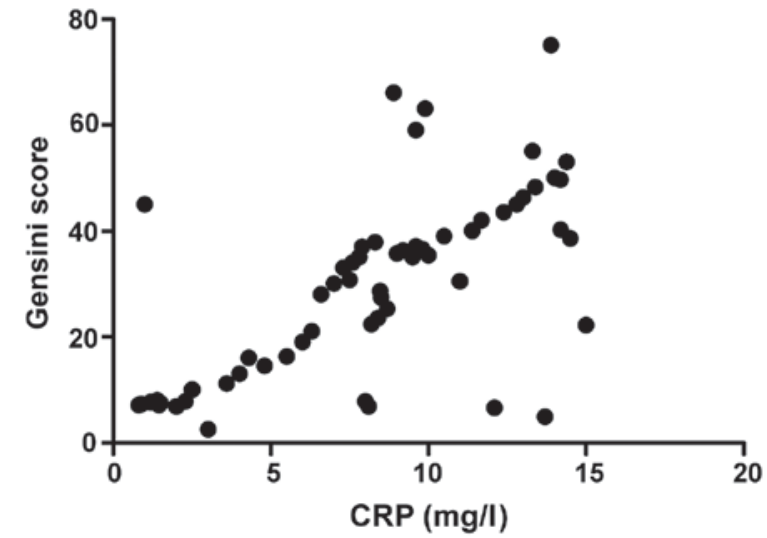

Figure 3. Correlation analysis between CRP and Gensini score. CRP, $\mathrm{C}$-reactive protein.

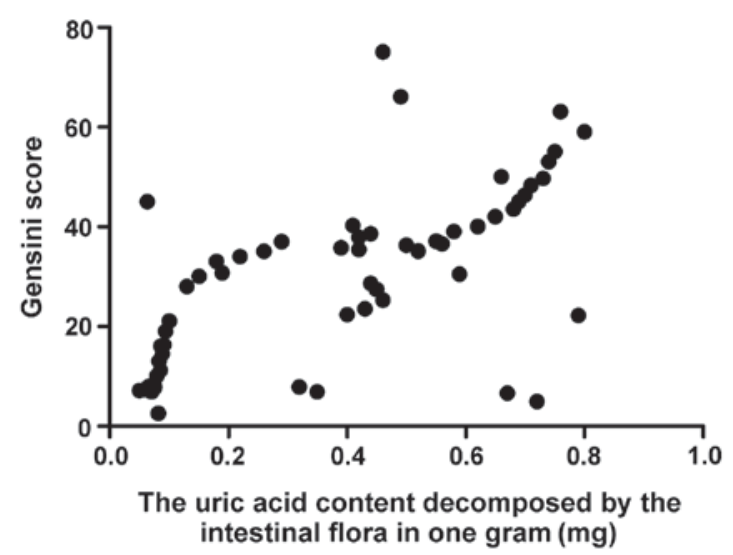

Figure 4. Correlation analysis between IFUA activity and Gensini score. IFUA, uric acid content decomposed by the intestinal flora.

FUA (16), and the increase of BUA level, which is consistent with the conclusion of the present study.

It was found in an in vitro study that a variety of Lactobacilli and Bifidobacteria were extracted from feces of normal people and concluded that these two strains had very strong effects, and the most obvious one was against Pseudomonas aeruginosa preventing the formation of pseudomembrane (17). When intestinal flora disturbance occurs in obese patients, the intestinal mucosa will be damaged, the body's inflammatory response is excessive, and the serum levels of CRP, IL-6 and TNF- $\alpha$ are elevated; atherosclerosis itself is an inflammatory disease (18). The increased levels of inflammatory factors lead to vascular endothelial damage, along with increased expression of adhesion molecules, disorders of vascular regulatory factor secretion, and vascular endothelial damage, eventually leading to atherosclerosis. It was also found in the present study that the levels of inflammatory factors in obese patients complicated with CHD were significantly higher than those in only obese patients, and the inflammatory factor levels were positively correlated with total intestinal bacterial load and Gensini score, indicating that when flora disturbance occurs in obese patients, the higher the inflammatory factor levels are, the greater the possibility of CHD will be $(19,20)$.

In conclusion, the occurrence of flora disturbance in obese patients may lead to CHD, and the levels of inflammatory 
factors and the level of uric acid decomposed by intestinal bacteria are also closely related to the occurrence and development process of the disease. The treatment of intestinal flora disturbance and reduction of inflammatory response can prevent or treat CHD to some extent.

\section{Acknowledgements}

Not applicable.

\section{Funding}

No funding was received.

\section{Availability of data and materials}

All data generated or analyzed during this study are included in this published article.

\section{Authors' contributions}

XL designed the study, CL collected and analysed the data, XL prepared the manuscript. All authors read and approved the final manuscript.

\section{Ethics approval and consent to participate}

This study was approved by the Ethics Committee of Maternity and Child Health Care of Zaozhuang (Zaozhuang, China). Signed informed consents were obtained from all patients or their guardians before the study.

\section{Consent for publication}

Not applicable.

\section{Competing interests}

The authors declare that they have no competing interests.

\section{References}

1. Yang T, Santisteban MM, Rodriguez V, Li E, Ahmari N, Carvajal JM, Zadeh M, Gong M, Qi Y, Zubcevic J, et al: Gut dysbiosis is linked to hypertension. Hypertension 65: 1331-1340, 2015.

2. Lau E, Carvalho D and Freitas P: Gut microbiota: Association with NAFLD and metabolic disturbances. BioMed Res Int 2015 $1-10,2015$.

3. Qin J, Li Y, Cai Z, Li S, Zhu J, Zhang F, Liang S, Zhang W, Guan Y, Shen D, et al: A metagenome-wide association study of gut microbiota in type 2 diabetes. Nature 490: 55-60, 2012.
4. Ley RE, Turnbaugh PJ, Klein S and Gordon JI: Microbial ecology: Human gut microbes associated with obesity. Nature 444: 1022-1023, 2006.

5. Wahlström A, Sayin SI, Marschall HU and Bäckhed F: Intestinal crosstalk between bile acids and microbiota and its impact on host metabolism. Cell Metab 24: 41-50, 2016.

6. Canfora EE, Jocken JW and Blaak EE: Short-chain fatty acids in control of body weight and insulin sensitivity. Nat Rev Endocrinol 11: 577-591, 2015.

7. Biedermann L and Rogler G: The intestinal microbiota: Its role in health and disease. Eur J Pediatr 174: 151-167, 2015.

8. Eckburg PB, Bik EM, Bernstein CN, Purdom E, Dethlefsen L, Sargent M, Gill SR, Nelson KE and Relman DA: Diversity of the human intestinal microbial flora. Science 308: 1635-1638, 2005.

9. Zhang J, Chen SL and Li LB: Correlation between intestinal flora and serum inflammatory factors in patients with Crohn's disease. Eur Rev Med Pharmacol Sci 21: 4913-4917, 2017.

10. Sargent J: Obesity: Rethinking inflammation and adipocyte homeostasis. Nat Rev Endocrinol 10: 446, 2014.

11. van Greevenbroek MM, Schalkwijk CG and Stehouwer CD: Obesity-associated low-grade inflammation in type 2 diabetes mellitus: Causes and consequences. Neth J Med 71: 174-187, 2013.

12. El Kaoutari A, Armougom F, Gordon JI, Raoult D and Henrissat B: The abundance and variety of carbohydrate-active enzymes in the human gut microbiota. Nat Rev Microbiol 11: 497-504, 2013.

13. Tang C, Ahmed K, Gille A, Lu S, Gröne HJ, Tunaru S and Offermanns S: Loss of FFA2 and FFA3 increases insulin secretion and improves glucose tolerance in type 2 diabetes. Nat Med 21: 173-177, 2015.

14. Kimura I, Ozawa K, Inoue D, Imamura T, Kimura K, Maeda T, Terasawa K, Kashihara D, Hirano K, Tani T, et al: The gut microbiota suppresses insulin-mediated fat accumulation via the short-chain fatty acid receptor GPR43. Nat Commun 4: 1829, 2013.

15. Kasai C, Sugimoto K, Moritani I, Tanaka J, Oya Y, Inoue H, Tameda M, Shiraki K, Ito M, Takei Y, et al: Comparison of the gut microbiota composition between obese and non-obese individuals in a Japanese population, as analyzed by terminal restriction fragment length polymorphism and next-generation sequencing. BMC Gastroenterol 15: 100, 2015.

16. Bishara J, Farah R, Mograbi J, Khalaila W, Abu-Elheja O, Mahamid $\mathrm{M}$ and Nseir W: Obesity as a risk factor for clostridium difficile infection. Clin Infect Dis 57: 489-493, 2013.

17. Wang H and Eckel RH: Lipoprotein lipase: From gene to obesity. Am J Physiol Endocrinol Metab 297: 271-288, 2009.

18. Kotzbeck P and Zechner R: Angiopoietin-like 4: An endogenous break of intestinal lipid digestion. Mol Metab 3: 88-89, 2014.

19. Bäckhed F, Manchester JK, Semenkovich CF and Gordon JI: Mechanisms underlying the resistance to diet-induced obesity in germ-free mice. Proc Natl Acad Sci USA 104: 979-984, 2007.

20. Mahalle N, Garg M, Kulkarni M and Naik S: Association of inflammatory cytokines with traditional and nontraditional cardiovascular risk factors in indians with known coronary artery disease. Ann Med Health Sci Res 4: 706-712, 2014.

This work is licensed under a Creative Commons Attribution-NonCommercial-NoDerivatives 4.0 International (CC BY-NC-ND 4.0) License. 